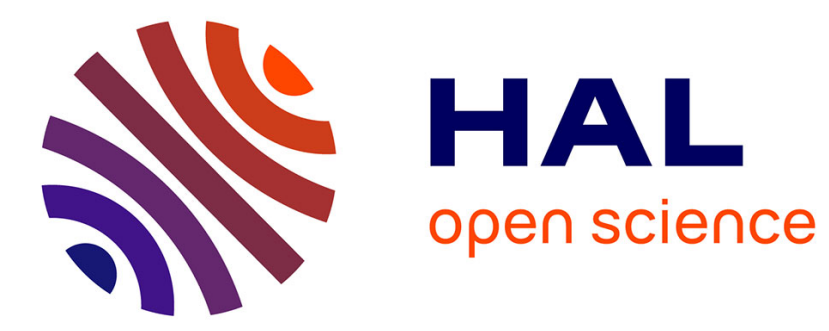

\title{
General relative entropy in a nonlinear McKendrick model
}

Philippe Michel

\section{To cite this version:}

Philippe Michel. General relative entropy in a nonlinear McKendrick model. Stochastic Analysis and Partial Differential Equations, pp.205 - 232, 2007, 978-0-8218-4059-7. 10.1090/conm/429/08238 . hal-01485052

\section{HAL Id: hal-01485052 https://hal.science/hal-01485052}

Submitted on 8 Mar 2017

HAL is a multi-disciplinary open access archive for the deposit and dissemination of scientific research documents, whether they are published or not. The documents may come from teaching and research institutions in France or abroad, or from public or private research centers.
L'archive ouverte pluridisciplinaire HAL, est destinée au dépôt et à la diffusion de documents scientifiques de niveau recherche, publiés ou non, émanant des établissements d'enseignement et de recherche français ou étrangers, des laboratoires publics ou privés. 


\title{
General Relative Entropy in a nonlinear McKendrick model
}

\author{
P. Michel
}

\begin{abstract}
We use the General Relative Entropy Inequality introduced in [12, $13,14]$ to analyze the long time convergence in a nonlinear renewal equation, a PDE that describes age structured populations for instance. More precisely, we prove that under some assumptions on the nonlinear term in a model of McKendrick-Von Foerster we deduce easily the global convergence using the General Relative Entropy (GRE) method of entropy. Then we compare the local asymptotic results obtained by linearisation and by the GRE principle.
\end{abstract}

\section{Introduction}

In the study of populations of cells, animals or humans, one of the most used model is the McKendrick-Von Foerster model $([\mathbf{9}, \mathbf{1 6}])$ where the density of population $n(t, y)$ at time $t$ and age $y$, is described by the master equation

$$
\left\{\begin{array}{l}
\frac{\partial}{\partial t} n(t, y)+\frac{\partial}{\partial y} n(t, y)+d(y) n(t, y)=0, \\
n(t, 0)=\int_{0}^{\infty} B\left(y^{\prime}\right) n\left(t, y^{\prime}\right) d y^{\prime},
\end{array}\right.
$$

where $B \geq 0$ is the birth rate, $d \geq 0$ is the death rate. It is well known that the long time asymptotic is described by the first eigenvalue $\lambda$ and positive eigenvector $N$ of the eigenproblem (1.2). Indeed, for a large time, $n \sim C s t N e^{\lambda t}$, this can be proved by using Laplace transform $([\mathbf{9}, \mathbf{5}])$ and more recently by using the General Relative Entropy (GRE) inequalities $([\mathbf{1 5}, \mathbf{1 4}, \mathbf{1 2}, \mathbf{1 0}])$. We recall the GRE results, if there exist $(N, \lambda, \phi)$ solution to the following eigenproblem

$$
\left\{\begin{array}{l}
\frac{\partial}{\partial y} N(y)+(d(y)+\lambda) N(y)=0 \\
N(0)=\int_{0}^{\infty} B\left(y^{\prime}\right) N\left(y^{\prime}\right) d y^{\prime}, \quad N>0 \\
-\frac{\partial}{\partial y} \phi(y)+(d(y)+\lambda) \phi(y)=B(y) \phi(0), \quad \phi \geq 0, \quad \phi \neq 0,
\end{array}\right.
$$

then for all positive convex function $H$ we have

$$
\frac{d}{d t} \int_{0}^{\infty} H\left(n(t, y) e^{-\lambda t} / N(y)\right) N(y) \phi(y) d y=-D_{H}\left(n e^{-\lambda t} / N\right) \leq 0,
$$

1991 Mathematics Subject Classification. 35Bxx.

Key words and phrases. General Relative Entropy, Nonlinear McKendrick model, Global convergence. 
with the dissipation of entropy $-D_{H}$ given by

$$
-D_{H}\left(n e^{-\lambda t} / N\right)=N(0) \phi(0)\left(H\left(\int_{0}^{\infty} \frac{n e^{-\lambda t}}{N} d \mu\right)-\int_{0}^{\infty} H\left(\frac{n e^{-\lambda t}}{N}\right) d \mu\right),
$$

where $d \mu(y)=B(y) N(y) d y / \int_{0}^{\infty} B\left(y^{\prime}\right) N\left(y^{\prime}\right) d y^{\prime}$ is a probability measure. In particular, for $H(x)=x$, we obtain the conservation of $\int_{0}^{\infty} n(t, y) e^{-\lambda t} \phi(y) d y$. The convergence of $n e^{-\lambda t}$ to $C s t N$ implies the exponential growth or extinction. This of course does not take in account the use of resources for instance. In order to take in account the consumption of nutrient, we can for instance change the linear birth term in $(1.1)$ by a nonlinear birth term $([\mathbf{2}, \mathbf{9}, \mathbf{1 7}])$

$$
\left\{\begin{array}{l}
\frac{\partial}{\partial t} n(t, y)+\frac{\partial}{\partial y} n(t, y)+d(y, P(t)) n(t, y)=0, \\
n(t, 0)=\int_{0}^{\infty} B\left(y^{\prime}, P(t)\right) n\left(t, y^{\prime}\right) d y^{\prime}, \\
P(t)=\int_{0}^{\infty} U\left(y^{\prime}\right) n\left(t, y^{\prime}\right) d y^{\prime},
\end{array}\right.
$$

where $P$ is related to the population number and the non linearities to the consumption of nutrients. Here we study the following nonlinear problem

$$
\left\{\begin{array}{l}
\frac{\partial}{\partial t} n(t, y)+\frac{\partial}{\partial y} n(t, y)+d(y) n(t, y)=0, \\
n(t, 0)=f\left(\int_{0}^{\infty} B\left(y^{\prime}\right) n\left(t, y^{\prime}\right) d y^{\prime}\right)
\end{array}\right.
$$

where $f$ describes the nonlinearity and so the change of birth term with the environment alteration, for example we can choose $f(x)=x^{\omega}$ with $0<\omega<1$. We notice that (1.6) is a particular case of the problem (1.5),

$$
\begin{gathered}
B\left(y^{\prime}, P(t)\right)=B\left(y^{\prime}\right) f(P(t)) / P(t), \\
d\left(y^{\prime}, P(t)\right)=d\left(y^{\prime}\right), \quad P(t)=\int_{0}^{\infty} B\left(y^{\prime}\right) n\left(t, y^{\prime}\right) d y^{\prime} .
\end{gathered}
$$

Even though this is not the most biologically relevant situation at least it is simple enough to classify the type of results we can obtain. There are several some theorems giving the local asymptotic behavior, i.e., in a neighbourhood of a steady state $[\mathbf{1}, \mathbf{4}, \mathbf{6}, \mathbf{7}, \mathbf{1 7}]$, in which, one uses the linearisation of (1.6), then checks for the eigenvector and eigenvalue of the linear system and according to the sign of the eigenvalue concludes to the local asymptotic convergence or not. Here we analyze how the GRE method can be used for this class of nonlinear problem and to the more general case

$$
\left\{\begin{array}{l}
\frac{\partial}{\partial t} n(t, y)+\frac{\partial}{\partial y} n(t, y)+d(y) n(t, y)=0 \\
n(t, 0)=f\left(\int_{0}^{\infty} B\left(y^{\prime}\right) n\left(t, y^{\prime}\right) d y^{\prime}, \int_{0}^{\infty} \theta\left(y^{\prime}\right) n\left(t, y^{\prime}\right) d y^{\prime}\right)
\end{array}\right.
$$


or for the following space-age model,

$$
\left\{\begin{aligned}
& \frac{\partial}{\partial t} n(t, y, x)+\frac{\partial}{\partial y}(V(y, x) n(t, y, x))+d(y, x) n(t, y, x) \\
&-\frac{\partial}{\partial x}\left[\nu(y, x) \frac{\partial}{\partial x} n(t, y, x)\right]=0 \\
& n(t, 0, x)=f\left(\int_{0}^{\infty} b\left(y^{\prime}, x\right) n\left(t, y^{\prime}, x\right) d y^{\prime}\right)
\end{aligned}\right.
$$

where $y$ is the age and $x$ the space parameter. As, we will see, in several situations we can arrive at global behaviors.

This paper is organized as follows : in section 2 we extend the definition of $(N, \lambda, \phi)$ to this nonlinear problem and prove the longtime convergence under some assumptions on $f$, especially we arrive to global pictures for extinction or attraction to a steady state. Then in section 4 we compare then results obtained by local asymptotic method and the GRE results for periodic or oscillating solutions. In section 6 we extend the result to (1.7) and (1.8).

\section{Nonlinear McKendrick}

First we define the eigenproblem, and in particular, the adjoint eigenproblem in the nonlinear equation (1.6), then we use the GRE introduce in (1.3) (see [14, 12, 10]). Thus, under global assumptions on $f$ we prove the decay of specific entropy and the global convergence in long time in (1.6). We notice that (1.6) can be written in a simpler way. We introduce the following notations that will be used throughout the paper

$$
\begin{gathered}
D(y):=\int_{0}^{y} d(s) d s, \\
g(y):=\beta f(y), \\
d \mu(y):=B(y) e^{-D(y)} d y / \beta,
\end{gathered}
$$

where, and this is a first assumption,

$$
\beta:=\int_{0}^{\infty} B\left(y^{\prime}\right) e^{-D\left(y^{\prime}\right)} d y^{\prime}<\infty .
$$

Notice that $d \mu($.$) is a probability measure. Then we set$

$$
m(t, y):=\beta n(t, y) e^{D(y)}
$$

and $m(t,$.$) satisfies the master equation in L^{p}\left(\left[0, \infty[) \bigcap L^{1}([0, \infty[, \phi(y) d y)\right.\right.$

$$
\left\{\begin{array}{l}
\frac{\partial}{\partial t} m(t, y)+\frac{\partial}{\partial y} m(t, y)=0 \\
m(t, 0)=g\left(\int_{0}^{\infty} m\left(t, y^{\prime}\right) d \mu\left(y^{\prime}\right)\right), \\
m(0, .) \in L_{+}^{\infty}\left(\left[0, \infty[) \cap L_{+}^{1}([0, \infty[, \phi(y) d y),\right.\right.
\end{array}\right.
$$

where $p>1$. From now, we study the behavior of $m$ and we give assumptions on $g$ in order to simplify the notations. We prove the existence of a decreasing GRE and the longtime convergence of $m$ under some assumptions on $g$. At this stage we cover two simple cases : global extinction, global attraction to a non zero steady state. 


\section{Eigenproblem in nonlinear case}

3.1. Extinction. As for the linear case, the GRE principle is based on the existence of an adjoint eigenfunction problem, here we focus on the case where the eigenvalue is zero, i.e., the adjoint steady state. We notice there always exists $\phi$ uniquely defined up to multiplicative constant, solution to

$$
-\frac{\partial}{\partial y} \phi=\phi(0) B(y) e^{-D(y)} / \beta, \quad \phi \geq 0, \quad \phi \not \equiv 0, \quad \phi(\infty)=0 .
$$

Indeed, the function

$$
\phi(y):=\phi(0) \int_{y}^{\infty} B\left(y^{\prime}\right) e^{-D\left(y^{\prime}\right)} d y^{\prime} / \beta=\phi(0) \int_{y}^{\infty} d \mu(y),
$$

is well defined because we have $\int_{0}^{\infty} d \mu(y)=1$ and satisfies (3.1).

Theorem 3.1. Assume (2.4), $m(0,.) \in L^{\infty} \cap L^{1}([0, \infty[, \phi(y) d y)$ for eq. (2.6) and

$$
g(x)<x, \quad g \quad \text { u.s.c. }
$$

then $N=0$ is a globally attractive steady state. We have

$$
\begin{aligned}
\int_{0}^{\infty} & m(t, y) \phi(y) d y \quad \text { decreases, } \\
& 0 \leq m(t, .) \leq\|m(0, .)\|_{\infty}, \\
& \lim _{t \rightarrow \infty} \int_{0}^{\infty} m(t, y) d \mu(y)=0 .
\end{aligned}
$$

Proof. We prove the convergence (3.6), using the equation (2.6) and (3.2), we have

$$
\begin{aligned}
& \frac{d}{d t} \int_{0}^{\infty} m(t, y) \phi(y) d y=\phi(0) m(t, 0)-\phi(0) \int_{0}^{\infty} m(t, y) d \mu(y), \\
& \frac{d}{d t} \int_{0}^{\infty} m(t, y) \phi(y) d y=\phi(0)\left[g\left(\int_{0}^{\infty} m(t, y) d \mu(y)\right)-\int_{0}^{\infty} m(t, y) d \mu(y)\right],
\end{aligned}
$$

using (3.3), we have,

$$
\frac{d}{d t} \int_{0}^{\infty} m(t, y) \phi(y) d y \leq 0 .
$$

We prove the convergence of $\int_{0}^{\infty} m(t, y) d \mu(y)$ to 0 using a method developped in [14].

Step1- $L^{\infty}$ bound. For all $C>0$, we have (as a particular case of a GRE)

$$
\begin{aligned}
\frac{d}{d t} \int_{0}^{\infty}(m(t, y)-C)_{+} \phi(y) d y & \\
& =\phi(0)(m(t, 0)-C)_{+}-\phi(0) \int_{0}^{\infty}(m(t, y)-C)_{+} d \mu(y)
\end{aligned}
$$


using (3.3) and Jensen inequality, we have,

$$
\begin{aligned}
\frac{d}{d t} \int_{0}^{\infty}(m(t, y)-C)_{+} \phi(y) d y= & \phi(0)\left[\left(g\left(\int_{0}^{\infty} m(t, y)-d \mu(y)\right)-C\right)_{+}\right. \\
& \left.-\int_{0}^{\infty}(m(t, y)-C)_{+} d \mu(y)\right] \leq 0 .
\end{aligned}
$$

Since $m(0,.) \in L^{\infty}([0, \infty[)$ (see $(2.6))$, we have

$$
m(t, y) \leq m(0, y), \quad \forall t, y \geq 0,
$$

and $m(t,$.$) is uniformly bounded in L^{\infty}([0, \infty[)$. Therefore, we may choose a sequence of times $t_{k} \rightarrow \infty$ so that the sequence of functions $m_{k}(t, y)=m\left(t+t_{k}, y\right)$ converges weak-* in $L^{\infty}([0, T] \times[0, \infty[)$, for any $T>0$, to a function denoted $\bar{m}(t, y)$.

Step2- $L^{1}$ bound. From (3.9), we have the decay of $\int_{0}^{\infty} m(t, y) \phi(y) d y$ and thus its convergence to a positive number. Now, using (3.8), we obtain

$$
\int_{0}^{\infty}\left[\int_{0}^{\infty} m(t, y) d \mu(y)-g\left(\int_{0}^{\infty} m(t, y) d \mu(y)\right)\right] d t \leq \int_{0}^{\infty} m(0, y) \phi(y) d y
$$

and thus

$$
\int_{0}^{T}\left[\int_{0}^{\infty} m_{k}(t, y) d \mu(y)-g\left(\int_{0}^{\infty} m_{k}(t, y) d \mu(y)\right)\right] d t \rightarrow 0 \quad \text { as } \quad k \rightarrow \infty .
$$

Step3-Compactness. For any smooth function $\psi(y)$, and using equation (2.6), we have that

$$
\frac{d}{d t} \int_{0}^{\infty} m_{k}(t, y) \psi(y) d y \text { is bounded in } L^{\infty}([0, \infty[) .
$$

Therefore, after approximating $B e^{-D}$ by smooth functions, we deduce that $\int_{0}^{\infty} m_{k}(t, y) d \mu(y)$ converges in $C([0, T])$ to $\int_{0}^{\infty} \bar{m}(t, y) d \mu(y)$. Now using that $g$ is u.s.c. and satisfies (3.3), we have

$$
0 \leq \int_{0}^{T}\left[\int_{0}^{\infty} \bar{m}(t, y) d \mu(y)-g\left(\int_{0}^{\infty} \bar{m}(t, y) d \mu(y)\right)\right] d t
$$

$$
\leq \liminf _{k \rightarrow \infty} \int_{0}^{T}\left[\int_{0}^{\infty} m_{k}(t, y) d \mu(y)-g\left(\int_{0}^{\infty} m_{k}(t, y) d \mu(y)\right)\right] d t=0,
$$

thus

$$
\int_{0}^{T}\left[\int_{0}^{\infty} \bar{m}(t, y) d \mu(y)-g\left(\int_{0}^{\infty} \bar{m}(t, y) d \mu(y)\right)\right] d t=0,
$$

but using (3.3), we have

$$
\lim _{t_{k} \rightarrow \infty} \int_{0}^{\infty} m\left(t+t_{k}, y\right) d \mu(y)=\int_{0}^{\infty} \bar{m}(t, y) d \mu(y)=0 .
$$

Using transport equation (2.6) with boundary condition that converges to 0 , we have the global convergence of $m(t,$.$) to 0$ in $L^{\infty}([0, \infty[)$.

TheOREm 3.2. Assume there exists $\lambda_{0}<0$ such that

$$
g(y) \leq \gamma y, \quad \text { with } \quad \gamma:=\frac{\beta}{\int_{0}^{\infty} B\left(y^{\prime}\right) e^{-D\left(y^{\prime}\right)-\lambda_{0} y^{\prime}} d y^{\prime}}>0
$$


then $N=0$ is a stable steady state and there exists $\phi$, uniquely defined up to a multiplicative constant, a solution to

$$
-\frac{\partial}{\partial y} \phi+\lambda_{0} \phi(y)=\phi(0) B(y) e^{-D(y)} / \int_{0}^{\infty} B\left(y^{\prime}\right) e^{-D\left(y^{\prime}\right)-\lambda_{0} y^{\prime}} d y^{\prime},
$$

and for this $\phi$ we have

$$
\int_{0}^{\infty} m\left(t, y^{\prime}\right) \phi\left(y^{\prime}\right) d y^{\prime} \leq e^{\lambda_{0} t} \int_{0}^{\infty} m\left(0, y^{\prime}\right) \phi\left(y^{\prime}\right) d y^{\prime} .
$$

Proof. The $\phi$ are given by the following formula :

$$
\phi(y):=\phi(0) \int_{y}^{\infty} B\left(y^{\prime}\right) e^{-D\left(y^{\prime}\right)-\lambda_{0}\left(y^{\prime}-y\right)} d y^{\prime} / \int_{0}^{\infty} B\left(y^{\prime}\right) e^{-D\left(y^{\prime}\right)-\lambda_{0} y^{\prime}} d y^{\prime},
$$

indeed $\phi$ is well defined (using $\int_{0}^{\infty} d \mu(y)=1$ ) and satisfies (3.18).

Now we prove the exponential convergence under assumption (3.17), we have, using $\phi$ defined by the equation (3.18)

$$
\begin{aligned}
& \frac{d}{d t} \int_{0}^{\infty} m(t, y) e^{-\lambda_{0} t} \phi(y) d y= \\
& \phi(0) e^{-\lambda_{0} t}\left[m(t, 0)-\frac{\beta}{\int_{0}^{\infty} B\left(y^{\prime}\right) e^{-D\left(y^{\prime}\right)-\lambda_{0} y^{\prime}} d y^{\prime}} \int_{0}^{\infty} m(t, y) d \mu(y)\right],
\end{aligned}
$$

using that $\lambda_{0}$ is defined by (3.17), we have,

$$
\frac{d}{d t} \int_{0}^{\infty} m(t, y) e^{-\lambda_{0} t} \phi(y) d y \leq 0,
$$

therefore we have (3.19).

3.2. Globally attractive steady state. Next we prove the existence of a steady state when $g$ has a fixed point and $d \mu$ satisfies

$$
\int_{0}^{\infty} y d \mu(y)<\infty
$$

and there exists $p>1$ s.t.

$$
\int_{\text {sup Supp } B} \frac{\left(B(y) e^{-D(y)}\right)^{p /(p-1)}}{\left(\int_{y}^{\infty} d \mu\left(y^{\prime}\right)\right)^{1 /(p-1)}} d y<\infty .
$$

REMARK 3.3. The condition (3.24) is satisfied for a large class of $B$ and $d$. a) If Supp $B$ is compact, $\max \operatorname{Supp} B=y_{B}$ and $B(y) \sim_{y \rightarrow y_{B}} C_{r}\left(y_{B}-y\right)^{r}$ where $0<$ $C_{r}<\infty$ and $-1<r<+\infty$ then (3.24) is satisfied for $p>\max (1,(r+2) /(r+1))$. b) If $B(y) e^{-D(y)} \leq C_{r} e^{-r y}$ where $0<C_{r}<\infty$ and $r>0$ then (3.24) is satisfied for all $p>1$.

More precisely we have

Lemma 3.4. Assume (3.23) and

$$
\exists x_{0}>0, \quad g\left(x_{0}\right)=x_{0},
$$


then there exists $N \geq 0(N \neq 0)$ such that $N\left(x_{0}\right)=x_{0}$ and

$$
\left\{\begin{array}{l}
\frac{\partial}{\partial y} N(y)=0, \\
N(0)=g\left(\int_{0}^{\infty} N\left(y^{\prime}\right) d \mu\left(y^{\prime}\right)\right) .
\end{array}\right.
$$

And there exists $\phi \geq 0, \phi \not \equiv 0$ that satisfies the following adjoint eigenproblem

$$
-\frac{\partial}{\partial y} \phi=\phi(0) B(y) e^{-D(y)}, \quad \int_{0}^{\infty} N(y) \phi(y) d y=1 .
$$

Proof. Indeed, let

$$
\begin{gathered}
N(y):=x_{0}, \\
\phi(y):=\phi(0) \int_{y}^{\infty} d \mu\left(y^{\prime}\right),
\end{gathered}
$$

then $N$ satisfies (3.26) and $\phi$ satisfies (3.27).

3.3. GRE and first results. Here we consider the relative entropy introduce in (1.3) and give assumptions on $g$ that allow us to use it. From now we assume there exist a strictly positive steady state and adjoint steady state solution $(N, \phi)$ to $(3.26),(3.27)$.

First we prove the existence of a GRE entropy structure in the nonlinear problem (2.6). Under some assumptions on $g$, we prove, the decay of entropy, some bounds on $m(t,$.$) and the convergence of m(t,$.$) to N($.$) .$

THEOREM 3.5. Under the assumption (2.4), with the notations of section 2, and these of lemma 3.4, let the entropy defined by

$$
\mathcal{H}(t):=\int_{0}^{\infty} H(m / N) N \phi d y
$$

with $N$ and $\phi$ solution to (3.26), (3.27) and $H$ a convex function. Then we have

$$
\begin{aligned}
\frac{\partial}{\partial t} \mathcal{H}(t) & =N(0) \phi(0) \\
& {\left[H\left(\frac{g\left(\int_{0}^{\infty} m\left(t, y^{\prime}\right) d \mu\left(y^{\prime}\right)\right)}{g(N(0))}\right)-\frac{\int_{0}^{\infty} H(m / N) N(y) d \mu(y)}{N(0)}\right], }
\end{aligned}
$$

and define equivalently $\left(N(y)=x_{0}\right)$

$$
\begin{aligned}
& -D_{H}(m / N)(t):=\frac{\partial}{\partial t} \mathcal{H}(t) \\
& 1)=N(0) \phi(0)\left[H\left(\frac{g\left(x_{0} \int_{0}^{\infty} \frac{m\left(t, y^{\prime}\right)}{N\left(y^{\prime}\right)} d \mu\left(y^{\prime}\right)\right)}{g\left(x_{0}\right)}\right)-\int_{0}^{\infty} H\left(\frac{m}{N}\right) d \mu(y)\right] .
\end{aligned}
$$

Proof. Indeed we have

$$
\begin{aligned}
\frac{\partial}{\partial t} \mathcal{H}(t)=\int_{0}^{\infty} H^{\prime}(m / N) \phi \frac{\partial}{\partial t} m(t, y) d y \\
=-\int_{0}^{\infty} H^{\prime}(m / N) \phi \frac{\partial}{\partial y} m(t, y) d y
\end{aligned}
$$


Figure 1. Condition of convergence.

$$
\begin{aligned}
\frac{\partial}{\partial t} \mathcal{H}(t)=-\int_{0}^{\infty} \frac{\partial}{\partial y}(H(m / N) \phi N) d y & +\int_{0}^{\infty} H(m / N) \frac{\partial}{\partial y}(\phi N) d y \\
& -\int_{0}^{\infty} H^{\prime}(m / N) \phi \frac{m}{N^{2}} \frac{\partial}{\partial y} N d y,
\end{aligned}
$$

and finally we obtain

$(3.34) \frac{\partial}{\partial t} \mathcal{H}(t)=(H(m / N) \phi N)(t, y=0)-\int_{0}^{\infty} H(m / N) N \phi(0) B e^{-D(y)} d y / \beta$,

$$
\frac{\partial}{\partial t} \mathcal{H}(t)=N(0) \phi(0)
$$

$$
\left[H\left(\frac{g\left(\int_{0}^{\infty} m\left(t, y^{\prime}\right) d \mu\left(y^{\prime}\right)\right)}{g(N(0))}\right)-\frac{\int_{0}^{\infty} H(m / N) N(y) d \mu(y)}{N(0)}\right],
$$

where $N(y)=N(0)=x_{0}$ and the conclusion follows.

Under some assumptions on $g$ (and thus on $f$, see (2.2)) we prove the decay of some entropy and the convergence of $m$ to $N$. More precisely,

$$
\left\{\begin{array}{l}
\exists x_{0}>0: \quad g\left(x_{0}\right)=x_{0}, \\
x<g(x)<x_{0}, \quad \forall x<x_{0}, \\
x_{0}<g(x)<x, \quad \forall x>x_{0} .
\end{array}\right.
$$

This implies directly the uniqueness of the fixed point of $g$ and thus the uniqueness of a strictly positive steady state of (1.6).

Remark 3.6. For instance (3.36) is satisfied for $g(x)=x^{\omega}$ with $0<\omega<1$ and more generally if $g$ and so $f$ is an increasing concave function. See Figure 1. (3.36).

Here we prove some $L^{\infty}$ a priori bounds, using theorem 3.5 and assumption

Lemma 3.7. Assume (2.4), (3.36) and $0 \leq m(0,.) \leq K N($.$) for some K \geq 1$ then

$$
0 \leq m(t, y) \leq K N(y), \quad \forall t, y \geq 0 .
$$

Proof. We have for $H(z)=(z-K)_{+}$, using (3.31) with $K \geq 1$,

$$
\begin{aligned}
& -D_{H}(m / N)(t)=N(0) \phi(0) \\
& \quad\left[\left(\frac{g\left(\int_{0}^{\infty} \frac{m\left(t, y^{\prime}\right)}{N\left(y^{\prime}\right)} d \mu\left(y^{\prime}\right) x_{0}\right)}{g\left(x_{0}\right)}-K\right)_{+}-\int_{0}^{\infty}\left(\frac{m}{N}-K\right)_{+} d \mu(y)\right],
\end{aligned}
$$


but using (3.36), we have

$$
\begin{aligned}
& -D_{H}(m / N)(t)=N(0) \phi(0) \\
& \quad\left[\left(\int_{0}^{\infty} \frac{m\left(t, y^{\prime}\right)}{N\left(y^{\prime}\right)} d \mu\left(y^{\prime}\right)-K\right)_{+}-\int_{0}^{\infty}\left(\frac{m}{N}-K\right)_{+} d \mu(y)\right] \leq 0 .
\end{aligned}
$$

Thus, $m(0,.) \leq K N($.$) , implies \int_{0}^{\infty}(m(t, y) / N(y)-K)_{+} N(y) \phi(y) d y=0$ for $t=0$, decreases and is positive thus

$$
\int_{0}^{\infty}(m(t, y) / N(y)-K)_{+} N(y) \phi(y) d y=0, \quad \forall t \geq 0,
$$

and the conclusion follows.

LEMma 3.8. Assume (2.4), (3.36) and $m(0,.) \geq k N($.$) for some 1 \geq k>0$ then

$$
m(t, y) \geq k N(y), \forall t, y \geq 0 .
$$

Proof. We have for $H(z)=(m-z)_{+}$, using (3.31) with $0<m \leq 1$,

$$
-D_{H}(m / N)(t)=N(0) \phi(0)
$$

$$
\left[\left(k-\frac{g\left(\int_{0}^{\infty} \frac{m\left(t, y^{\prime}\right)}{N\left(y^{\prime}\right)} d \mu\left(y^{\prime}\right) x_{0}\right)}{g\left(x_{0}\right)}\right)_{+}-\int_{0}^{\infty}\left(k-\frac{m}{N}\right)_{+} d \mu(y)\right],
$$

but using (3.36) we have

$$
\begin{aligned}
& -D_{H}(m / N)(t)=N(0) \phi(0) \\
& \qquad\left[\left(k-\int_{0}^{\infty} \frac{m\left(t, y^{\prime}\right)}{N\left(y^{\prime}\right)} d \mu\left(y^{\prime}\right)\right)_{+}-\int_{0}^{\infty}\left(k-\frac{m}{N}\right)_{+} d \mu(y)\right] \leq 0 .
\end{aligned}
$$

Thus, $m(0,.) \geq k N($.$) , implies \int_{0}^{\infty}(k-m(t, y) / N(y))_{+} N(y) \phi(y) d y=0$ for $t=0$, decreases and is positive thus

$$
\int_{0}^{\infty}(k-m(t, y) / N(y))_{+} N(y) \phi(y) d y=0, \quad \forall t \geq 0,
$$

and the conclusion follows.

Now we prove the following convergence result,

Theorem 3.9. Assume (2.4), (3.23), (3.24), (3.36),

$$
m(0, .) \in L^{1}\left(\left[0, \infty[, \phi(y) d y) \bigcap L^{\bar{p}}([0, \infty[, \phi(y) d y), \quad \bar{p}>p,\right.\right.
$$

with $p$ defined in (3.24) and $\int_{0}^{\infty} m\left(0, y^{\prime}\right) \phi\left(y^{\prime}\right) d y^{\prime}>0$, then

$$
\begin{gathered}
\underline{I}:=\inf _{t \geq 0} \int_{0}^{\infty} m\left(t, y^{\prime}\right) \phi\left(y^{\prime}\right) d y^{\prime}>0, \\
\bar{I}:=\sup _{t \geq 0} \int_{0}^{\infty} m\left(t, y^{\prime}\right) \phi\left(y^{\prime}\right) d y^{\prime}<\infty .
\end{gathered}
$$

Moreover, we have

$$
\lim _{t \rightarrow \infty} m(t, .) / N(.)=1, \quad L^{1}\left(\left[0, \infty\left[, N\left(y^{\prime}\right) \phi\left(y^{\prime}\right) d y^{\prime}\right) .\right.\right.
$$


THEOREM 3.10. We make the assumptions of theorem 3.9, for some $\alpha>0$,

$$
\frac{d \mu(y)}{d y}=B(y) e^{-D(y)} / \beta \geq \alpha \phi(y) / \phi(0), \quad \forall y \geq 0,
$$

$$
J:=\min \left(1-\sup _{1 \leq z \leq \max (1, \bar{J})}\left(\frac{\frac{g\left(z x_{0}\right)}{g\left(x_{0}\right)}-1}{z-1}\right), \inf _{\min (1, \underline{J}) \leq z \leq 1}\left(\frac{\frac{g\left(z x_{0}\right)}{g\left(x_{0}\right)}-1}{z-1}\right)-1\right)>0,
$$

where

$$
\underline{J}:=\inf _{t \geq 0} \int_{0}^{\infty} m\left(t, y^{\prime}\right) d \mu\left(y^{\prime}\right) \geq 0, \quad \bar{J}:=\sup _{t \geq 0} \int_{0}^{\infty} m\left(t, y^{\prime}\right) d \mu\left(y^{\prime}\right) \leq \infty
$$

Then

$$
\begin{aligned}
& \int_{0}^{\infty} \mid m\left(t, y^{\prime}\right)- N\left(y^{\prime}\right) \mid \phi\left(y^{\prime}\right) d y^{\prime} \leq \\
& e^{-J \alpha N(0) \phi(0) t} \int_{0}^{\infty}\left|m\left(0, y^{\prime}\right)-N\left(y^{\prime}\right)\right| \phi\left(y^{\prime}\right) d y^{\prime}
\end{aligned}
$$

REMARK 3.11. We notice that when $g$ is $C^{1} \frac{\frac{g\left(z x_{0}\right)}{g\left(x_{0}\right)}-1}{z-1}$ gives the slope of $g$, and $J>0$ means that the slope of $g$ is strictly less than 1 at the right of the fixed point $x_{0}$ and strictly greater than 1 at the left of $x_{0}$. Moreover (3.49) can be replaced by

$$
\underline{J}:=\inf _{t \geq t_{0}} \int_{0}^{\infty} m\left(t, y^{\prime}\right) \frac{B\left(y^{\prime}\right) e^{-D\left(y^{\prime}\right)} d y^{\prime}}{\beta} \geq 0,
$$

for some $t_{0}>0$, the same for (3.49) and then appears a constant $C\left(t_{0}\right)$ in the right hand side of (3.50).

ProOF. of theorem 3.9 First we prove (3.45), (3.44) then we prove the convergence result (3.46).

Step1. Bound (3.45). Under the assumptions (3.36) and lemmas 3.7 and 3.8, the dissipation of entropy associated to $H(z)=|1-z|$ satisfies,

$$
-D_{H}=-D_{|1-z|}=-D_{(1-z)_{+}}-D_{(1-z)_{-}} \leq 0,
$$

and

$$
\int_{0}^{\infty}|m(t, y)-N(y)| \phi(y) d y \leq \int_{0}^{\infty}|m(0, y)-N(y)| \phi(y) d y<\infty,
$$

thus using $m(0, y) \in L^{1}([0, \infty[, \phi(y) d y)$ and (3.23), we obtain,

$$
\begin{aligned}
& \int_{0}^{\infty} m(t, y) \phi(y) d y \leq \\
& \quad \int_{0}^{\infty} N(y) \phi(y) d y+\int_{0}^{\infty}|m(0, y)-N(y)| \phi(y) d y<\infty, \quad \forall t \geq 0 .
\end{aligned}
$$


Step2. Bound $L^{\bar{p}}$. Under the assumptions (3.36) and adapting lemmas 3.7 and 3.8 , the dissipation of entropy associated to $H(z)=|1-z|^{\bar{p}}$ satisfies,

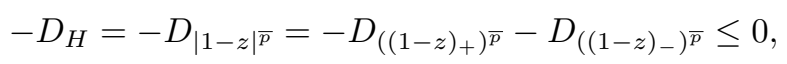

and

$$
\int_{0}^{\infty}(m(t, y)-N(y))^{\bar{p}} \phi(y) d y \leq \int_{0}^{\infty}(m(0, y)-N(y))^{\bar{p}} \phi(y) d y<\infty
$$

thus using (3.43), (3.23) and (3.45), we obtain,

$$
\begin{aligned}
& \int_{0}^{\infty} m(t, y)^{\bar{p}} \phi(y) d y \leq\left[\left(\int_{0}^{\infty} N^{\bar{p}}(y) \phi(y) d y\right)^{1 / \bar{p}}\right. \\
& \left.\quad+\left(\int_{0}^{\infty}(m(0, y)-N(y))^{\bar{p}} \phi(y) d y\right)^{1 / \bar{p}}\right]^{\bar{p}}<\infty, \quad \forall t \geq 0
\end{aligned}
$$

Moreover we have the uniform $L^{\bar{p}}([0, \infty[, \phi(y) d y)$ bound

$$
M_{\bar{p}}:=\sup _{t \geq 0} \int_{0}^{\infty} m(t, y)^{\bar{p}} \phi(y) d y<\infty .
$$

Step3. Bound (3.44). Since Supp B $\subset$ Supp $\phi$ and using the Hölder inequality with $1 / p+1 / q=1,1<p \leq \bar{p}$ and (3.24), we find

$$
\begin{aligned}
\int_{0}^{\infty} m(t, y) & d \mu(y)=\int_{0}^{\infty} m(t, y) B(y) e^{-D(y)} / \beta d y \\
& =\int_{\text {supp } \phi} m(t, y) \phi(y)^{1 / p} \frac{B(y) e^{-D(y)}}{\beta \phi(y)^{1 / p}} d y \\
\leq & \left(\int_{0}^{\infty} m(t, y)^{p} \phi(y) d y\right)^{1 / p}\left(\int_{\text {supp } \phi} \frac{\left(B(y) e^{-D(y)}\right)^{q}}{\beta^{q} \phi(y)^{q / p}} d y\right)^{1 / q} \\
\leq & K(p)\left(\int_{0}^{\infty} m(t, y)^{p} \phi(y) d y\right)^{1 / p},
\end{aligned}
$$

with

$$
K(p):=\left(\int_{\text {supp } \phi} \frac{\left(B(y) e^{-D(y)}\right)^{p /(p-1)}}{\beta^{p /(p-1)} \phi(y)^{1 /(p-1)}} d y\right)^{(p-1) / p}<\infty .
$$

Now, we use the Hölder inequality for $1 / p+1 / q=1,1<p \leq \bar{p}$ and we obtain

$$
\begin{gathered}
\int_{0}^{\infty} m(t, y) \phi(y) d y \leq\left(\int_{0}^{\infty} m(t, y)^{p} \phi(y) d y\right)^{1 / p}\left(\int_{0}^{\infty} \phi(y) d y\right)^{1 / q} \\
=\left(\int_{0}^{\infty} y d \mu(y)\right)^{1 / q}\left(\int_{0}^{\infty} m(t, y)^{p} \phi(y) d y\right)^{1 / p}
\end{gathered}
$$

Similarly, the Hölder inequality with $1 / p^{\prime}+1 / q^{\prime}=1, q^{\prime}:=\frac{\bar{p}-1}{p-1}>1$ implies

$$
\begin{aligned}
\int_{0}^{\infty} m^{p}(t, y) \phi(y) d y=\int_{0}^{\infty} m^{1 / p^{\prime}} m^{p-1 / p^{\prime}}(t, y) \phi(y) d y \\
\leq\left(\int_{0}^{\infty} m(t, y) \phi(y) d y\right)^{1 / p^{\prime}}\left(\int_{0}^{\infty} m^{\left(p-1 / p^{\prime}\right) q^{\prime}}(t, y) \phi(y) d y\right)^{1 / q^{\prime}} \\
\leq\left(\int_{0}^{\infty} m(t, y) \phi(y) d y\right)^{1 / p^{\prime}}\left(\int_{0}^{\infty} m^{\left(p-1+1 / q^{\prime}\right) q^{\prime}}(t, y) \phi(y) d y\right)^{1 / q^{\prime}} \\
\quad \leq\left(\int_{0}^{\infty} m(t, y) \phi(y) d y\right)^{1 / p^{\prime}}\left(\int_{0}^{\infty} m^{\bar{p}}(t, y) \phi(y) d y\right)^{1 / q^{\prime}}
\end{aligned}
$$


P. MICHEL

Therefore, using (3.57) and (3.23), for all $1<p<\bar{p}$, we have

$$
\begin{aligned}
& \underline{C}_{p}\left(\int_{0}^{\infty} m(t, y) \phi(y) d y\right)^{\frac{p}{p-1}} \\
& \quad \leq \int_{0}^{\infty} m^{p}(t, y) \phi(y) d y \leq \bar{C}_{p}\left(\int_{0}^{\infty} m(t, y) \phi(y) d y\right)^{\frac{\bar{p}-p}{p}-1}
\end{aligned}
$$

where

$$
\underline{C}_{p}:=\left(\int_{0}^{\infty} y d \mu(y)\right)^{-\frac{1}{p-1}}<\infty
$$

and

$$
\bar{C}_{p}:=M_{\bar{p}}^{\frac{p-1}{\bar{p}-1}}<\infty
$$

Thus, using (3.36) and (3.31) for $H(z)=z$, we have,

$$
\frac{d}{d t} \int_{0}^{\infty} m(t, y) \phi(y) d y>0, \quad \text { when } \quad \int_{0}^{\infty} m(t, y) d \mu(y)<1
$$

and so

$$
\frac{d}{d t} \int_{0}^{\infty} m(t, y) \phi(y) d y>0
$$

when $\int_{0}^{\infty} m(t, y) \phi(y) d y<\bar{C}_{p}^{\frac{1-\bar{p}}{\bar{p}-p}} K(p)^{\frac{p(\bar{p}-1)}{\bar{p}-p}}:=C$, and (3.44) follows with $\underline{I} \geq$ $\min \left(C, \int_{0}^{\infty} m(0, y) \phi(y) d y\right)>0$.

Step4. Convergence. Moreover, for $H(z)=|1-z|^{p}, D_{H}=0$ if and only if $m / N$ is a constant $\rho(t)$, indeed using (3.58) and (3.61), we have

$$
\sup _{t \geq 0} \int_{0}^{\infty} m(t, y) d \mu(y)<\infty
$$

and

$$
\begin{aligned}
& -D_{|1-z|^{p}}(m / N)(t) \leq N(0) \phi(0) \\
& \quad\left[\left|1-\int_{0}^{\infty} \frac{m(t, y)}{N(y)} d \mu(y)\right|^{p}-\int_{0}^{\infty}\left|1-\frac{m}{N}\right|^{p} d \mu(y)\right],
\end{aligned}
$$

thus $m / N=\rho$ independent of $y$. Moreover if $\rho(t) N$ satisfies (1.6) and (3.44), with assumption (3.36) that implies uniqueness of the strictly positive steady state then $\rho(t)=1$. Using the transport equation with bounds given by lemma 3.8 and 3.7 , we have for all $t_{k} \nearrow \infty, m\left(t+t_{k},.\right) / N($.$) has a weak L^{\infty}([0, \infty[, d \mu)$ limit, by passing to the limit in the dissipation of entropy and in the equation (1.6) (we refer to $[\mathbf{1 4}]$ of to the proof of lemma 3.1 to the complete proof), we have $\lim _{k \rightarrow \infty} m\left(t+t_{k},.\right) / N()=$.1 .

Proof. of theorem 3.10 Now, we prove the exponential decay. We recall that for all $H$ positive convex function we have

$$
\begin{aligned}
\frac{\partial}{\partial t} \int_{0}^{\infty} H(m / N) N \phi d y=N(0) \phi(0) & \\
& {\left[H\left(\frac{g\left(\int_{0}^{\infty} \frac{m\left(t, y^{\prime}\right)}{N\left(y^{\prime}\right)} d \mu\left(y^{\prime}\right) x_{0}\right)}{g\left(x_{0}\right)}\right)-\int_{0}^{\infty} H\left(\frac{m}{N}\right) d \mu(y)\right], }
\end{aligned}
$$


thus using (3.49) we have

$$
\begin{aligned}
& \frac{\partial}{\partial t} \int_{0}^{\infty}(m / N-1)_{+} N \phi d y= \\
& N(0) \phi(0)\left[\left(\frac{g\left(\int_{0}^{\infty} \frac{m\left(t, y^{\prime}\right)}{N\left(y^{\prime}\right)} d \mu\left(y^{\prime}\right) x_{0}\right)}{g\left(x_{0}\right)}-1\right)_{+}-\int_{0}^{\infty}\left(\frac{n}{N}-1\right)_{+} d \mu(y)\right] \\
& \leq N(0) \phi(0)\left[\gamma_{+}\left(\int_{0}^{\infty} \frac{m\left(t, y^{\prime}\right)}{N\left(y^{\prime}\right)} d \mu\left(y^{\prime}\right)-1\right)_{+}-\int_{0}^{\infty}\left(\frac{m}{N}-1\right)_{+} d \mu(y)\right],
\end{aligned}
$$

with $\gamma_{+}=\sup _{1 \leq x \leq \max (1, \bar{J})}\left(\frac{g\left(x x_{0}\right) / g\left(x_{0}\right)-1}{x-1}\right)$ and (3.47) implies

$$
\begin{aligned}
& \frac{\partial}{\partial t} \int_{0}^{\infty}(m / N-1)_{+} N \phi d y \leq \\
& \alpha\left(\gamma_{+}-1\right) \int_{0}^{\infty}(m / N-1)_{+} N \phi d y .
\end{aligned}
$$

In the same way, using (3.49) we have

$$
\begin{aligned}
& \frac{\partial}{\partial t} \int_{0}^{\infty}(m / N-1)_{-} N \phi d y= \\
& N(0) \phi(0)\left[\left(\frac{g\left(\int_{0}^{\infty} \frac{m\left(t, y^{\prime}\right)}{N\left(y^{\prime}\right)} d \mu\left(y^{\prime}\right) x_{0}\right)}{g\left(x_{0}\right)}-1\right)_{-}-\int_{0}^{\infty}\left(\frac{m}{N}-1\right)_{-} d \mu(y)\right] \\
& \leq N(0) \phi(0)\left[\gamma_{-}\left(\int_{0}^{\infty} \frac{m\left(t, y^{\prime}\right)}{N\left(y^{\prime}\right)} d \mu\left(y^{\prime}\right)-1\right)_{-}-\int_{0}^{\infty}\left(\frac{m}{N}-1\right)_{-} d \mu(y)\right],
\end{aligned}
$$

with $\gamma_{-}=\inf _{\min (\underline{J}, 1) \leq x \leq 1}\left(\frac{g\left(x x_{0}\right) / g\left(x_{0}\right)-1}{x-1}\right)$ and (3.47) implies

$$
\frac{\partial}{\partial t} \int_{0}^{\infty}(m / N-1)_{-} N \phi d y \leq \alpha\left(1-\gamma_{-}\right) \int_{0}^{\infty}(m / N-1)_{-} N \phi d y .
$$

Therefore (3.50) follows from Gronwall lemma.

This theorem gives results if we have some informations such as the conservation law given by the linear case for instance or the uniqueness of the steady state in the nonlinear case.

REMARK 3.12. We notice that the condition

$$
\begin{cases}1<g\left(z x_{0}\right) / g\left(x_{0}\right)<2-z, & \forall z<1 \\ 1>g\left(z x_{0}\right) / g\left(x_{0}\right)>2-z, & \forall z>1\end{cases}
$$

is satisfied for $g(x)=\min \left(x^{\omega}, a\right)$ with $\frac{\ln (2-a)}{\ln (a)}<\omega<0,0<a<1$, and implies the convergence to $N$ as in the theorem 3.9. 
P. MICHEL

3.4. Blow-up. Here, we give some conditions on $g$ that imply the blow-up of $m$ solution to $(2.6)$.

Theorem 3.13. Assume (2.4) and there exist $\gamma>1, \alpha>0$ such that

$$
\begin{gathered}
g(x) \geq \gamma x, \\
\frac{d \mu(y)}{d y}=B(y) e^{-D(y)} / \beta \geq \alpha \phi(y) / \phi(0), \quad \forall y \geq 0,
\end{gathered}
$$

then we have

$$
\int_{0}^{\infty} m(t, y) \phi(y) d y>e^{\alpha(\gamma-1) t} \int_{0}^{\infty} m(0, y) \phi(y) d y .
$$

Proof. We prove the convergence (3.67), using the equation (2.6) and (3.2) introduced in section 3.1, we have

$$
\begin{gathered}
\frac{d}{d t} \int_{0}^{\infty} m(t, y) \phi(y) d y=\phi(0) m(t, 0)-\phi(0) \int_{0}^{\infty} m(t, y) d \mu(y) \\
\frac{d}{d t} \int_{0}^{\infty} m(t, y) \phi(y) d y=\phi(0)\left[g\left(\int_{0}^{\infty} m(t, y) d \mu(y)\right)-\int_{0}^{\infty} m(t, y) d \mu(y)\right]
\end{gathered}
$$

using (3.3), we have,

$$
\frac{d}{d t} \int_{0}^{\infty} m(t, y) \phi(y) d y>\phi(0)(\gamma-1) \int_{0}^{\infty} m(t, y) d \mu(y),
$$

and finally

$$
\frac{d}{d t} \int_{0}^{\infty} m(t, y) \phi(y) d y>\alpha(\gamma-1) \int_{0}^{\infty} m(t, y) \phi(y) d y .
$$

Thus, using Gronwall inequality, we obtain (3.67) and

$$
\lim _{t \rightarrow \infty} \int_{0}^{\infty} m(t, y) \phi(y) d y=\infty .
$$

Now, it is interesting to compare this result to the linearisation method in order to prove the local asymptotic convergence.

\section{Local asymptotic convergence}

In this section we compare the linearisation and GRE results in the case where there exists only one $x_{0}>0$ such that $g\left(x_{0}\right)=x_{0}$, i.e, there exists one and only one steady state different from 0 . Now we give some assumptions on $g$ such that the strictly positive steady state $N$ of the equation (1.6) be locally asymptotically stable. We begin with conditions for the stability (instability) of the linearized problem then we prove a sharp result on the nonlinear problem.

Theorem 4.1. (Linearisation method) Assume there exists an unique $x_{0}>0$ such that $g\left(x_{0}\right)=x_{0}$ and

$$
\left.\int_{0}^{\infty} e^{-r y^{\prime}} d \mu\left(y^{\prime}\right)<\infty, \quad \forall r \in\right]-\infty, \infty[,
$$


then the steady state associated to $x_{0}, N(y)=x_{0}$ is a locally asymptotically stable steady state when

$$
0<\left|g^{\prime}\left(x_{0}\right)\right|<1
$$

and $N(y)=x_{0}$ is a locally asymptotically unstable steady state when

$$
g^{\prime}\left(x_{0}\right)>1 .
$$

Proof. In a neighbourhood of the steady state we set $m=N+\tilde{\epsilon}$ and

$$
\left\{\begin{array}{l}
\frac{\partial}{\partial t} \tilde{\epsilon}(t, y)+\frac{\partial}{\partial y} \tilde{\epsilon}(t, y)=0, \\
\tilde{\epsilon}(t, 0)=g^{\prime}\left(x_{0}\right) \int_{0}^{\infty} \tilde{\epsilon}\left(t, y^{\prime}\right) d \mu\left(y^{\prime}\right)+O\left(\sup _{y} \tilde{\epsilon}^{2}(t, y)\right) .
\end{array}\right.
$$

Therefore $m$ solution to (1.6) converges locally asymptotically to the steady state $N$ if the eigenvalue associated to the system is strictly negative (see $[\mathbf{1}, \mathbf{4}, \mathbf{6}, \mathbf{7}, \mathbf{1 7}]$ ). Using the General Relative Entropy in the linear case, and the approximation $\tilde{\epsilon} \sim \epsilon$ where $\epsilon$ satisfies the linear problem

$$
\left\{\begin{array}{l}
\frac{\partial}{\partial t} \epsilon(t, y)+\frac{\partial}{\partial y} \epsilon(t, y)=0, \\
\epsilon(t, 0)=g^{\prime}\left(x_{0}\right) \int_{0}^{\infty} \epsilon\left(t, y^{\prime}\right) d \mu\left(y^{\prime}\right),
\end{array}\right.
$$

we deduce the asymptotic behavior of $\epsilon$.

Step1. $0<\left|g^{\prime}\left(x_{0}\right)\right|<1$. In this case, there exists $(\lambda, \bar{\phi})$ solution to

$$
\left\{\begin{array}{l}
-\frac{\partial}{\partial y} \bar{\phi}(y)+\lambda \bar{\phi}(y)=\bar{\phi}(0)\left|g^{\prime}\left(x_{0}\right)\right| B(y) e^{-} D(y) / \beta, \\
\bar{\phi}(y) \geq 0, \quad \forall y \geq 0 .
\end{array}\right.
$$

Indeed, using (4.1), there exists $\lambda<0$ s.t.

$$
\int_{0}^{\infty} e^{-\lambda y^{\prime}} d \mu\left(y^{\prime}\right)=\left|1 / g^{\prime}\left(x_{0}\right)\right|
$$

and thus, for all $\bar{\phi}(0)>0, \bar{\phi}$ is uniquely defined and

$$
\bar{\phi}(y)=\bar{\phi}(0)\left|g^{\prime}\left(x_{0}\right)\right| \int_{y}^{\infty} e^{-\lambda\left(y^{\prime}-y\right)} d \mu\left(y^{\prime}\right) .
$$

Then, we use the GRE and we find

$$
\frac{d}{d t} \int_{0}^{\infty}\left|\epsilon(t, y) e^{-\lambda t}\right| \bar{\phi}(y) d y:=-D_{H}(t),
$$

where

$$
\begin{aligned}
& -D_{H}(t)=\phi(0)\left|g^{\prime}\left(x_{0}\right)\right| \\
& \quad\left[\left|\int_{0}^{\infty} \epsilon(t, y) e^{-\lambda t} d \mu(y)\right|-\int_{0}^{\infty}\left|\epsilon(t, y) e^{-\lambda t}\right| d \mu(y)\right] \leq 0,
\end{aligned}
$$

Therefore $\lambda<0$ and using the decay of GRE we have

$$
\int_{0}^{\infty}|\epsilon(t, y)| \bar{\phi}(y) d y \leq e^{\lambda t} \int_{0}^{\infty}|\epsilon(0, y)| \bar{\phi}(y) d y
$$

(and thus $\epsilon$ converges to 0 ) when (4.2) is satisfied. 
Step2. $g^{\prime}\left(x_{0}\right)>1$. In this case, there exists $(\bar{\epsilon}, \lambda, \bar{\phi})$ solution to

$$
\left\{\begin{array}{l}
\frac{\partial}{\partial y} \bar{\epsilon}(y)+\lambda \bar{\epsilon}(y)=0 \\
\bar{\epsilon}(0)=g^{\prime}\left(x_{0}\right) \int_{0}^{\infty} \bar{\epsilon}\left(y^{\prime}\right) d \mu\left(y^{\prime}\right), \\
-\frac{\partial}{\partial y} \bar{\phi}(y)+\lambda \bar{\phi}(y)=\bar{\phi}(0) g^{\prime}\left(x_{0}\right) B(y) e^{-} D(y) / \beta \\
\bar{\epsilon}(y), \quad \bar{\phi}(y) \geq 0, \quad \forall y \geq 0, \quad \int_{0}^{\infty} \bar{\epsilon}\left(y^{\prime}\right) \bar{\phi}\left(y^{\prime}\right) d y^{\prime}=1 .
\end{array}\right.
$$

Indeed, using (4.1), we have,

$$
\begin{gathered}
\int_{0}^{\infty} e^{-\lambda y^{\prime}} d \mu\left(y^{\prime}\right)=1 / g^{\prime}\left(x_{0}\right), \\
\bar{\phi}(y)=\bar{\phi}(0) g^{\prime}\left(x_{0}\right) \int_{y}^{\infty} e^{-\lambda\left(y^{\prime}-y\right)} d \mu(y) .
\end{gathered}
$$

Then, using the GRE, we obtain, for all $\rho \in]-\infty, \infty[$,

$$
\frac{d}{d t} \int_{0}^{\infty}\left|\epsilon(t, y) e^{-\lambda t}-\rho \bar{\epsilon}(y)\right| \bar{\phi}(y) d y:=-D_{H}(t),
$$

where

$$
\begin{aligned}
-D_{H}(t)=\phi(0) g^{\prime}\left(x_{0}\right)\left[\mid \int_{0}^{\infty}\right. & \left(\epsilon(t, y) e^{-\lambda t}-\rho \bar{\epsilon}(y)\right) d \mu(y) \mid \\
& \left.-\int_{0}^{\infty}\left|\epsilon(t, y) e^{-\lambda t}-\rho \bar{\epsilon}(y)\right| d \mu(y)\right] \leq 0 .
\end{aligned}
$$

Therefore $\lambda>0$ and using the decay of GRE when $g^{\prime}>1$, we obtain the convergence result

$$
\lim _{t \rightarrow \infty}\left\|\epsilon(t, .) e^{-\lambda t}-\rho \bar{\epsilon}(.)\right\|_{L^{1}([0, \infty[, d \mu)}=0,
$$

where $\rho$ is given by the conservation law

$$
\int_{0}^{\infty} \epsilon(t, y) e^{-\lambda t} \bar{\phi}(y) d y=\int_{0}^{\infty} \epsilon(0, y) \bar{\phi}(y) d y
$$

and thus $\epsilon$ does not converges to 0 .

Theorem 4.2. Assume there exist $\eta>0$ and an unique $x_{0}>0$ such that $g(x)=x_{0}$ for all $\left.x \in\right] x_{0}-\eta, x_{0}+\eta\left[\right.$ then the steady state associated to $x_{0}, N(y)=x_{0}$ is locally asymptotically stable.

Proof. Indeed, in this case $m(t, 0)=g\left(\int_{0}^{\infty} m(t, y) d \mu(y)\right)=x_{0}$ when $m(t,$. is in a neigborhood of $N()=.x_{0}$, therefore $N()=.x_{0}$ is locally asymptotically stable.

REMARK 4.3. Notice that (3.36) or the inequalities given in remark 3.12 give weaker locally conditions in a neighborhood of the steady state than the condition (4.2). In other words theorem 4.1 gives a weaker convergence than the theorems 3.9, 3.10 but needs weaker assumptions on $g$ than the theorems 3.9, 3.10. 
Now we prove that under some assumptions on $g$ the conditions (3.36) are equivalent to the condition (4.2).

LEMMA 4.4.

Assume $g$ belongs to $C^{1}([0, \infty[)$ is increasing and strictly concave, and there exists only one strictly positive solution to $g\left(x_{0}\right)=x_{0}$ then condition (3.36) is equivalent to $0<g^{\prime}\left(x_{0}\right)<1$.

Using the proof of theorem 3.9 we prove there exists some bounds on $n(t=$ $0,$.$) that gives the local exponential convergence. As we see it in section 5$ the following result is sharp because for 'large variations' of the initial data, there can be oscillations.

Theorem 4.5. Assume (3.66) for $\alpha>0, g$ belongs to $C^{1}([0, \infty[)$, and there exists an $x_{0}>0$ such that $g\left(x_{0}\right)=x_{0}$ and

$$
0<g^{\prime}\left(x_{0}\right)<1,
$$

then there exists $\eta_{+}, \eta_{-}>0$ such that

$$
\begin{aligned}
& \left.x<g(x)<x_{0}, \quad \forall x \in\right] x_{0}\left(1-\eta_{-}\right), x_{0}[, \\
& \left.x_{0}<g(x)<x, \quad \forall x \in\right] x_{0}, x_{0}\left(1+\eta_{+}\right)[.
\end{aligned}
$$

and

$$
I:=\min \left(1-\sup _{1 \leq z \leq 1+\eta_{+}} g^{\prime}\left(z x_{0}\right), \inf _{1-\eta_{-} \leq z \leq 1} g^{\prime}\left(z x_{0}\right)-1\right)>0 .
$$

Also for all $m(0,$.$) that satisfies$

$$
|m(0, .) / N(.)-1|_{\infty}<\eta:=\min \left(\eta_{+}, \eta_{-}\right),
$$

we have

$$
|m(t, .) / N(.)-1|_{\infty}<\eta, \quad \forall t \geq 0,
$$

and the local exponential convergence holds,

$$
\int_{0}^{\infty}|m(t, y)-N(y)| \phi(y) d y \leq e^{-I \alpha N(0) \phi(0) t} \int_{0}^{\infty}|m(0, y)-N(y)| \phi(y) d y .
$$

Proof. The inequalities (4.9), (4.10) are just taylor formula. Now, we prove the $L^{\infty}$ bound then we use the proof of theorem 3.9 to conclude.

Step1. Bound (4.13). Let $r(t, y)=m(t, y) / N(y)$ then using the transport equation, we have,

$$
r(t, y)=m(0, y) / N(y) 1_{t<y}+\left(\frac{g\left(x_{0} \int_{0}^{\infty} r\left(t-y, y^{\prime}\right) d \mu\left(y^{\prime}\right)\right)}{g\left(x_{0}\right)}-1\right) 1_{y \leq t},
$$

and $r(t, y):=L(r)(t, y)$. But $m(0, y) / N(y) \in\left\{f:|f-1|_{\infty}<\eta\right\}$ and

$$
L:\left\{f:|f-1|_{\infty}<\eta\right\} \longrightarrow\left\{f:|f-1|_{\infty}<\eta\right\},
$$

implies that $r \in\left\{f:|f-1|_{\infty}<\eta\right\}$ for all $t \geq 0$. 
Step2. Convergence. Then we adapt the proof of theorem 3.9, noticing that in this case $\bar{J} \leq 1+\eta_{+}, \underline{J} \geq 1-\eta_{-}$and $J$ defined in (3.48) is equal to

$$
J=\min \left(1-\sup _{1 \leq z \leq 1+\eta_{+}}\left(\frac{\int_{1}^{z} g^{\prime}\left(s x_{0}\right) d s}{z-1}\right), \inf _{1-\eta_{-} \leq z \leq 1}\left(\frac{\int_{1}^{z} g^{\prime}\left(s x_{0}\right) d s}{z-1}\right)-1\right)>0,
$$

thus $I \leq J$ and (4.14) follows.

Remark 4.6. Notice that when $g$ belongs to $C^{1}([0, \infty[)$, is convex and decreasing, and there exists only one strictly positive solution to $g\left(x_{0}\right)=x_{0}$ then the inequalities given in remark 3.12 are equivalent to $-1<g^{\prime}\left(x_{0}\right)<0$.

\section{Examples of oscillating solutions}

In this part we give some examples where oscillations appear, i.e., there is no convergence of the solution to a steady state.

5.1. Only unstable steady states. When all the steady states are unstable and then a bounded solution to (1.6) is bounded then the solution to (1.6) can oscillate. We see in the proof of theorem 4.1 and theorem 4.2 that, in the neighborhood of the steady state $N$, the solution behaves as $N+\epsilon$ with $\epsilon(t,.) \sim \bar{\epsilon}(.) e^{\lambda t}$ with $\lambda$ given by

$$
\int_{0}^{\infty} e^{-\lambda y^{\prime}} d \mu\left(y^{\prime}\right)=\left|1 / g^{\prime}\left(x_{0}\right)\right|
$$

when $\left|g^{\prime}\left(x_{0}\right)\right|<1$ or $g^{\prime}\left(x_{0}\right)>1$. Now, we are interested in the case where $g^{\prime}\left(x_{0}\right)<-1$. The existence of oscillating solution needs more information on $d \mu$,

$$
\left\{\begin{array}{l}
g\left(x_{0}\right)=x_{0}, \quad g^{\prime}\left(x_{0}\right)<-1, \\
p_{0}=\sup _{R e(p)}\left\{p \in C: \mathcal{B}(p)=1 / g^{\prime}\left(x_{0}\right)\right\}, \quad \operatorname{Re}\left(p_{0}\right) \geq 0 .
\end{array}\right.
$$

Lemma 5.1. Assume (5.1) then

$$
\epsilon(t, 0)=C e^{p_{0} t}(1+\Omega(t))
$$

where

$$
\lim _{t \rightarrow \infty} \Omega(t)=0
$$

and $x_{0}$ is an unstable steady state.

Proof. We refer to $[\mathbf{5}, \mathbf{7}]$ for the proof of this lemma which is based on the Laplace Transform.

We give an example of $g$ such that all its steady states are 'unstable' and an example of $d \mu$ satisfying (5.1).

EXAMPLE 5.2. Let $g(z)=\kappa z(1-z)_{+}$then the only steady states are $\{0,1-1 / \kappa\}$ and

$$
g^{\prime}(z)=\kappa(1-z)_{+}-\kappa z \operatorname{sgn}_{+}(1-z)
$$

and $g^{\prime}(0)=\kappa, g^{\prime}(1-1 / \kappa)=2-\kappa$ and all the steady states are 'unstable' when $\kappa>3$.

The existence of a solution to (5.1) is not automatic. For instance, let $d \mu(y)=$ $1_{[0,1]}(y) d y$ and $g^{\prime}\left(x_{0}\right)=-2$ then (5.1) is not satisfied and $\epsilon$ converges to 0 . 
EXAMPLE 5.3. Let $d \mu(y)=1_{[0,1]}(y) d y$ and $g^{\prime}\left(x_{0}\right)=-\kappa$ where $\kappa$ is large enough then (5.1) is satisfied and $\epsilon$ oscillates. Indeed, it suffices to prove that

$$
\left(1-e^{-p}\right) / p=-1 / \kappa,
$$

have almost a solution with a strictly positive real part. Let $p=a+i b$, with $a, b \in]-\infty, \infty[,(a, b) \neq(0,0)$, then (5.4) becomes

$$
e^{-a}=-\frac{b}{\kappa \sin (b)},
$$

and

$$
e^{-a}=\frac{1}{\cos (b)}\left(1-\frac{1}{\kappa} \ln \left(-\frac{b}{\kappa \sin (b)}\right)\right) .
$$

For all $\kappa>1$, there exists $b \in] 3 \pi / 2,2 \pi[$ such that

$$
-\frac{b}{\kappa \sin (b)}=\frac{1}{\cos (b)}\left(1-\frac{1}{\kappa} \ln \left(-\frac{b}{\kappa \sin (b)}\right)\right),
$$

and for $\kappa$ large enough $0<-\frac{b}{\kappa \sin (b)}<1$ and $a=-\ln \left(-\frac{b}{\kappa \sin (b)}\right)>0$.

TheOREm 5.4. Assume $g$ is bounded and all its fixed points satisfy

$$
\left|g^{\prime}\left(x_{0}\right)\right|>1
$$

and $d \mu$ satisfies (5.1) then if $m(0,.) \notin\{$ fixed points of $g\}$ the solution cannot converge to a steady state.

Proof. Indeed, the solution of (2.6) exists and is bounded, more precisely we have

$$
m(t, y) \leq \sup g(z), \quad \forall t<y,
$$

and $m(t, y)=m(y-t), \forall t \geq y$. Now, assume that $m(t,$.$) converges in L^{1}([0, \infty[, d \mu)$ to $\bar{m}($.$) then \bar{m}$ is a fixed point of $g$. But in a neighborhood of $\bar{m}$, we have $|m-\bar{m}| \sim$ $\bar{\epsilon}(.) e^{\lambda \bar{m} t}$ with $\lambda_{\bar{m}}>0$ and thus $\|m-\bar{m}\|_{L^{1}([0, \infty[, d \mu)}$ cannot converge to 0 .

5.2. At most one unstable steady state. We focus on the cases where there exist almost one unstable steady state, for instance, let $d=0, B(y)=(1 / 2) 1_{y \in[2,4]}$ and $g(x)=2$ if $x \geq 1, g(x)=0$ if $x<1$ then

$$
m(t, y)=2 \sum_{i=-\infty}^{\infty} 1_{y-t \in[3 i, 3 i+1]},
$$

satisfies

$$
\frac{\partial}{\partial t} m(t, y)+\frac{\partial}{\partial y} m(t, y)=0
$$

in distributional sense and

$$
\begin{aligned}
& \int_{0}^{\infty} m(t, y) B(y) d y=(1 / 2) \int_{2}^{4} m(t, y) d y \\
&=\sum_{i=-\infty}^{\infty} \int_{2}^{4} 1_{t-y \in[3 i, 3 i+1]} d y=\sum_{i=-\infty}^{\infty} \int_{t-4}^{t-2} 1_{y \in[3 i, 3 i+1]} d y
\end{aligned}
$$

but

$$
\int_{t-4}^{t-2} 1_{y \in[3 i, 3 i+1]} d y \geq 1 \quad \text { iff } \quad[t-4, t-2] \supset[3 i, 3 i+1]
$$


FigURE 2. transport of a periodic $m(t, y)$

and

$$
\sum_{i=-\infty}^{\infty} \int_{t-4}^{t-2} 1_{y \in[3 i, 3 i+1]} d y \geq 1 \quad \text { iff } \quad \exists i_{0} \quad \text { s.t. }[t-4, t-2] \supset\left[3 i_{0}, 3 i_{0}+1\right] .
$$

Thus we have

$$
\begin{gathered}
g\left(\sum_{i=-\infty}^{\infty} \int_{t-4}^{t-2} 1_{y \in[3 i, 3 i+1]} d y\right)=2 \quad \text { iff } \quad \exists i_{0}, \quad \text { s.t. }[t-4, t-2] \supset\left[3 i_{0}, 3 i_{0}+1\right], \\
g\left(\sum_{i=-\infty}^{\infty} \int_{t-4}^{t-2} 1_{y \in[3 i, 3 i+1]} d y\right)=0 \quad \text { iff } \quad \forall i_{0}[t-4, t-2] \text { not contain }\left[3 i_{0}, 3 i_{0}+1\right],
\end{gathered}
$$

finally we obtain that

$$
\begin{gathered}
g\left(\sum_{i=-\infty}^{\infty} \int_{t-4}^{t-2} 1_{y \in[3 i, 3 i+1]} d y\right)=2 \sum_{i_{0}=-\infty}^{\infty} 1_{[t-4, t-2] \supset\left[3 i_{0}, 3 i_{0}+1\right]}, \\
g\left(\sum_{i=-\infty}^{\infty} \int_{t-4}^{t-2} 1_{y \in[3 i, 3 i+1]} d y\right)=2 \sum_{i_{0}=-\infty}^{\infty} 1_{t \in\left[3 i_{0}, 3 i_{0}+1\right]},
\end{gathered}
$$

and

$$
g\left(\int_{0}^{\infty} m(t, y) B(y) d y\right)=m(t, 0)
$$

is a periodic solution that oscillates between the stable steady states 0 and 2 . See Figure 2.

Here we prove that under the assumption of linearity of $g$ around the unstable steady state and a condition over the Fourier transform of $B e^{-D}$,

$$
\mathcal{F}\left(B e^{-D}\right)=\int_{-\infty}^{\infty} B e^{-D}(y) 1_{y \geq 0} e^{-i \xi y} d y
$$

the unstable steady state generates a periodic solution to (1.6) (see [14]).

LEMMA 5.5.

Assume $g$ is linear in a neighbourhood of the unstable steady state, i.e., if $n=N+\epsilon$ with $\epsilon$ small enough then

$$
g\left(\int_{0}^{\infty} n\left(t, y^{\prime}\right) d \mu\left(y^{\prime}\right)\right)=g^{\prime}\left(\int_{0}^{\infty} N\left(y^{\prime}\right) d \mu\left(y^{\prime}\right)\right) \int_{0}^{\infty} \epsilon\left(y^{\prime}\right) d \mu\left(y^{\prime}\right),
$$

and the Fourier transform of $g^{\prime}\left(\int_{0}^{\infty} B\left(y^{\prime}\right) N\left(y^{\prime}\right) d y^{\prime}\right) B(y) e^{-D(y)} / \beta$ given by

$$
R(\xi):=\mathcal{F}\left(g^{\prime}\left(\int_{0}^{\infty} B\left(y^{\prime}\right) N\left(y^{\prime}\right) d y^{\prime}\right) B(y) e^{-D(y)} / \beta\right)(\xi),
$$

satisfies

$$
\exists \xi_{0}: \quad R\left(\xi_{0}\right)=1,
$$

then there exists an oscillating solution in a neighbourhood of the unstable steady state. 
Figure 3. Example of $g$

Proof. Let $n=N+\epsilon$

$$
\left\{\begin{array}{l}
\frac{\partial}{\partial t} \epsilon(t, y)+\frac{\partial}{\partial y} \epsilon(t, y)=0, \\
\epsilon(t, 0)=g^{\prime}\left(\int_{0}^{\infty} N\left(y^{\prime}\right) d \mu\left(y^{\prime}\right)\right) \int_{0}^{\infty} \epsilon\left(y^{\prime}\right) d \mu\left(y^{\prime}\right),
\end{array}\right.
$$

then let $\epsilon(t)=\tilde{\epsilon}(t-y)$, we have

$$
\begin{gathered}
\tilde{\epsilon}(t)=g^{\prime}\left(\int_{0}^{\infty} N\left(y^{\prime}\right) d \mu\left(y^{\prime}\right)\right) \int_{0}^{\infty} \tilde{\epsilon}\left(t-y^{\prime}\right) B\left(y^{\prime}\right) e^{-D\left(y^{\prime}\right)} d y^{\prime} / \beta, \\
\mathcal{F}(\tilde{\epsilon})(\xi)=R(\xi) \mathcal{F}(\tilde{\epsilon})(\xi),
\end{gathered}
$$

and

$$
\mathcal{F}(\tilde{\epsilon})(\xi)(1-R(\xi))
$$

thus using (5.8), we obtain that $\mathcal{F}(\tilde{\epsilon})(\xi)=\eta \delta_{\xi_{0}}(\xi), \eta$ small enough, is a periodic solution.

5.3. Only one locally stable steady state. The following example gives a local asymptotic convergence, i.e., a stable steady state in the case when $g$ satisfies (4.2) and does not satisfy (3.36), and which has oscillating solutions. This proves that the smallness assumptions, in theorem 4.2 and so, in the Theorem 4.5 is necessary. We notice that the limit case where $B(y)=\delta_{y=1}$ gives the simplest oscillating solutions, indeed, for all function $J$ such that

$$
J(t)=g(J(t-1))
$$

then

$$
m(t, y)=J(t-y)
$$

is a solution to (2.6). Moreover, for all $g$ that satisfies (4.2), $g(1)=1=x_{0}$, $g(1+\epsilon)=1-\epsilon$ and $g(1-\epsilon)=1+\epsilon$, with $0<\epsilon<1$, then one can take for instance

$$
J(t):=1+\epsilon \sum_{k=-\infty}^{\infty}(-1)^{k} 1_{t \in] k, k+1[},
$$

that gives a periodic solution. The following theorem gives an example of periodic solution for a $g$ such that the only strictly positive steady state 1 is locally asymptotically stable and nevertheless there exists some oscillating solution. Let $g$ satisfies

$$
\begin{gathered}
g(x)=\bar{g}>(1+\eta) /(1-\eta), \quad \forall x<1-\eta, \\
g(x)=0, \quad \forall x>1+\eta, \\
g(x)=1, \quad 1-\eta \leq x \leq 1+\eta,
\end{gathered}
$$

TheOrem 5.6. Assume (5.10)-(5.12) for $0<\eta<1 / 2$ and

$$
\text { Supp } B=K \subset[\alpha, \beta] \text {, }
$$

with $\infty>\alpha, \beta>0$ then for (see Figure 3)

$$
\epsilon_{0}=2 \eta / \bar{g}
$$




$$
n(0, y)=\bar{g} 1_{[0, \alpha]}(y)+1_{\left[\alpha, \alpha+\epsilon_{0}\right]}(y), \quad y \leq \beta,
$$

there exists $T>0$ such that

$$
n(T, y)=\bar{g} 1_{[0, \alpha]}(y)+1_{\left[\alpha, \alpha+\epsilon_{0}\right]}(y), \quad y \leq \beta .
$$

Proof. The idea of the proof is simple, we use the transport equation and its boundary condition to prove that after some times $t_{1}, t_{2}, t_{3}, t_{4}, T$ the solution comes back to the initial state on $y \in[0, \beta]$. Indeed, consider the initial data (5.15).

For $t_{1}=\epsilon_{1}$, such that,

$$
\epsilon_{0}+\bar{g} \epsilon_{1}=1-\eta
$$

we have

$$
n\left(t_{1}, y\right)=\bar{g} 1_{\left[0, \alpha+\epsilon_{1}\right]}(y)+1_{\left[\alpha+\epsilon_{1}, \alpha+\epsilon_{1}+\epsilon_{0}\right]}(y), \quad y \leq \beta .
$$

For $t_{2}=t_{1}+\epsilon_{2}$, such that,

$$
\epsilon_{0}+\bar{g} \epsilon_{1}+\bar{g} \epsilon_{2}=1+\eta
$$

we have

$$
n\left(t_{2}, y\right)=1_{\left[0, \epsilon_{2}\right]}+\bar{g} 1_{\left[\epsilon_{2}, \alpha+\epsilon_{1}+\epsilon_{2}\right]}(y)+1_{\left[\alpha+\epsilon_{1}+\epsilon_{2}, \alpha+\epsilon_{0}+\epsilon_{1}+\epsilon_{2}\right]}(y), \quad y \leq \beta .
$$

For $t_{3}=t_{2}+\beta-\epsilon_{3}$, such that,

$$
\epsilon_{2}+\bar{g} \epsilon_{3}=1+\eta
$$

we have

$$
n\left(t_{3}, y\right)=1_{\left[\beta-\epsilon_{3}-\epsilon_{2}, \beta-\epsilon_{3}\right]}+\bar{g} 1_{\left[\beta-\epsilon_{3}, \beta\right]}(y), \quad y \leq \beta .
$$

for $t_{4}=t_{3}+\epsilon_{4}$, such that,

$$
\epsilon_{2}+\bar{g} \epsilon_{3}-\bar{g} \epsilon_{4}=1-\eta,
$$

we have

$$
n\left(t_{4}, y\right)=1_{\left[0, \epsilon_{4}\right]}+1_{\left[\beta-\epsilon_{4}-\epsilon_{2}, \beta-\epsilon_{4}\right]}+\bar{g} 1_{\left[\beta-\epsilon_{4}, \beta\right]}(y), \quad y \leq \beta .
$$

Finally, for $T=t_{4}+\beta-\epsilon_{4}$, we have

$$
n(T, y)=\bar{g} 1_{[0, \alpha]}(y)+1_{\left[\alpha, \alpha+\epsilon_{4}\right]}(y), \quad y \leq \beta .
$$

Using (5.21), (5.23), we have $\epsilon_{4}=2 \eta / \bar{g}$ and thus using (5.14), we have $\epsilon_{0}=\epsilon_{4}$ and $n(T, y)=n(0, y)$ for all $y \leq \beta$.

\section{Generalization}

In this section we extend the GRE to the problem (1.7) and (1.8), using the same idea developed in section 4 . 


\subsection{Generalized age model (1.7). We define}

$$
\begin{gathered}
\beta_{0}:=\int_{0}^{\infty} B(y) N(y) d y, \\
\beta_{1}:=\int_{0}^{\infty} \theta\left(y^{\prime}\right) N\left(y^{\prime}\right) d y^{\prime}, \\
d \mu_{B}(y):=B(y) N(y) d y / \int_{0}^{\infty} B\left(y^{\prime}\right) N\left(y^{\prime}\right) d y^{\prime},
\end{gathered}
$$

and

$$
d \mu_{\theta}\left(y^{\prime}\right):=\theta(y) N(y) d y / \int_{0}^{\infty} \theta\left(y^{\prime}\right) N\left(y^{\prime}\right) d y^{\prime} .
$$

TheOREm 6.1. Assume there exist a positive steady state to the problem (1.7) and its dual,

$$
\left\{\begin{array}{l}
\frac{\partial}{\partial y} N(y)+d(y) N(t, y)=0, \\
N(0)=f\left(\int_{0}^{\infty} B(y) N(y) d y, \int_{0}^{\infty} \theta(y) N(y) d y\right), \\
-\frac{\partial}{\partial y} \phi(y)+d(y) \phi(y)=\phi(0) \chi(y), \quad \phi \geq 0,
\end{array}\right.
$$

for some function $\chi \geq 0$. Then for all positive convex function $H$, we have,

$$
\frac{\partial}{\partial t} \int_{0}^{\infty} H(n(t, y) / N(y)) N(y) \phi(y) d y:=-D_{H}(n / N),
$$

with

$$
\begin{aligned}
& -D_{H}(n / N)=N(0) \phi(0)[ \\
& \left.\quad H\left(\frac{f\left(\beta_{0} \int_{0}^{\infty} \frac{n}{N} d \mu_{B}(y), \beta_{1} \int_{0}^{\infty} \frac{n}{N} d \mu_{\theta}\left(y^{\prime}\right)\right)}{f\left(\beta_{0}, \beta_{1}\right)}\right)-\int_{0}^{\infty} H\left(\frac{n}{N}\right) \frac{\chi(y) N(y)}{N(0)} d y\right] .
\end{aligned}
$$

Proof. Indeed, we have,

$$
\begin{aligned}
& \frac{\partial}{\partial t} \int_{0}^{\infty} H(n(t, y) / N(y)) N(y) \phi(y) d y= \\
& \int_{0}^{\infty} H^{\prime}(n(t, y) / N(y)) \frac{\partial}{\partial t} n(t, y) \phi(y) d y,
\end{aligned}
$$




$$
\begin{aligned}
\frac{\partial}{\partial t} \int_{0}^{\infty} H(n(t, y) / N(y)) N(y) \phi(y) d y= \\
\quad-\int_{0}^{\infty} H^{\prime}(n(t, y) / N(y)) d(y) n(t, y) \phi(y) d y \\
\quad-\int_{0}^{\infty} \frac{\partial}{\partial y}[H(n(t, y) / N(y)) N(y) \phi(y)] d y \\
\quad+\int_{0}^{\infty} H(n(t, y) / N(y)) \frac{\partial}{\partial y}[N(y) \phi(y)] d y \\
\quad-\int_{0}^{\infty} H^{\prime}(n(t, y) / N(y)) \frac{n(t, y)}{N(y)^{2}} \phi(y) \frac{\partial}{\partial y} N(y) d y
\end{aligned}
$$

$$
\begin{aligned}
& \frac{\partial}{\partial t} \int_{0}^{\infty} H(n(t, y) / N(y)) N(y) \phi(y) d y= \\
& -\int_{0}^{\infty} H^{\prime}(n(t, y) / N(y)) d(y) n(t, y) \phi(y) d y \\
& +H(n(t, 0) / N(0)) N(0) \phi(0)-\int_{0}^{\infty} H(n(t, y) / N(y))[N(y) \phi(0) \chi(y)] d y \\
& +\int_{0}^{\infty} H^{\prime}(n(t, y) / N(y)) \phi(y) d(y) n(t, y) d y .
\end{aligned}
$$

Thus we have

$$
\begin{array}{r}
-D_{H}(n / N)=N(0) \phi(0)\left[H\left(\frac{f\left(\int_{0}^{\infty} n\left(t, y^{\prime}\right) B\left(y^{\prime}\right) d y^{\prime}, \int_{0}^{\infty} n\left(t, y^{\prime}\right) \theta\left(y^{\prime}\right) d y^{\prime}\right)}{f\left(\int_{0}^{\infty} N\left(y^{\prime}\right) B\left(y^{\prime}\right) d y^{\prime}, \int_{0}^{\infty} N\left(y^{\prime}\right) \theta\left(y^{\prime}\right) d y^{\prime}\right)}\right)\right. \\
\left.-\int_{0}^{\infty} H\left(\frac{n}{N}\right) \frac{\chi(y) N(y)}{N(0)} d y\right]
\end{array}
$$

and using (6.1)-(6.2) we conclude the proof of the theorem.

Now, we return to the longtime asymptotic. Assumption (3.36) is modified as

$$
\left\{\begin{array}{l}
\exists\left(\alpha_{0}, \alpha_{1}\right): 1=\frac{f\left(x x_{0}, y y_{0}\right)}{f\left(x_{0}, y_{0}\right)}, \quad \forall(x, y): \frac{\alpha_{0} x+\alpha_{1} y}{\alpha_{0}+\alpha_{1}}=1, \\
1<\frac{f\left(x x_{0}, y y_{0}\right)}{f\left(x_{0}, y_{0}\right)}<\frac{\alpha_{0} x+\alpha_{1} y}{\alpha_{0}+\alpha_{1}}, \quad \forall(x, y): \frac{\alpha_{0} x+\alpha_{1} y}{\alpha_{0}+\alpha_{1}}>1, \\
\frac{\alpha_{0} x+\alpha_{1} y}{\alpha_{0}+\alpha_{1}}<\frac{f\left(x x_{0}, y y_{0}\right)}{f\left(x_{0}, y_{0}\right)}<1, \quad \forall(x, y): \frac{\alpha_{0} x+\alpha_{1} y}{\alpha_{0}+\alpha_{1}}<1,
\end{array}\right.
$$

and we have the following theorem.

Theorem 6.2. Assume (6.7) and let

$$
\frac{\chi N(y)}{N(0)} d y=\left(\alpha_{0} d \mu_{B}(y)+\alpha_{1} d \mu_{\theta}(y)\right) /\left(\alpha_{0}+\alpha_{1}\right) \geq 0,
$$

then the entropy associated to $H(z)=|z-1|$ is decreasing and $n(t,.) \rightarrow N($.$) in$ $L^{1}([0, \infty[\times[0, \infty[, N(x, y) \phi(x, y) d x d y)$. 
Proof. Using the theorem 6.1 and the equation (6.7), with $H_{+}(z)=(z-1)_{+}$ and $H_{-}(z)=(z-1)_{-}$, we obtain

$$
\begin{array}{r}
-D_{H_{+}}(n / N)=N(0) \phi(0)\left[\left(\frac{f\left(\beta_{0} \int_{0}^{\infty} \frac{n}{N} d \mu_{B}(y), \beta_{1} \int_{0}^{\infty} \frac{n}{N} d \mu_{\theta}(y)\right)}{f\left(\beta_{0}, \beta_{1}\right)}-1\right)_{+}\right. \\
\left.-\int_{0}^{\infty}\left(\frac{n}{N}-1\right)_{+} \frac{\chi(y) N(y)}{N(0)} d y\right] .
\end{array}
$$

Using (6.7), we find

$$
\begin{array}{r}
-D_{H_{+}}(n / N) \leq N(0) \phi(0)\left[\left(\frac{\alpha_{0} \int_{0}^{\infty} \frac{n}{N} d \mu_{B}(y)+\alpha_{1} \int_{0}^{\infty} \frac{n}{N} d \mu_{\theta}(y)}{\alpha_{0}+\alpha_{1}}-1\right)_{+}\right. \\
\left.-\int_{0}^{\infty}\left(\frac{n}{N}-1\right)_{+} \frac{\chi(y) N(y)}{N(0)} d y\right],
\end{array}
$$

but (6.8) implies that

$$
\begin{aligned}
-D_{H_{+}}(n / N) \leq N(0) \phi(0) & \\
& {\left[\left(\int_{0}^{\infty} \frac{n}{N} \chi(y) d y(y)-1\right)_{+}-\int_{0}^{\infty}\left(\frac{n}{N}-1\right)_{+} \frac{\chi(y) N(y)}{N(0)} d y\right] . }
\end{aligned}
$$

Proceeding analogously, we find that

$$
\begin{aligned}
-D_{H_{-}}(n / N) \leq N(0) \phi(0) & \\
& {\left[\left(\int_{0}^{\infty} \frac{n}{N} \chi(y) d y(y)-1\right)_{-}-\int_{0}^{\infty}\left(\frac{n}{N}-1\right)_{-} \frac{\chi(y) N(y)}{N(0)} d y\right] . }
\end{aligned}
$$

The proof follows that of the theorem 3.9 and we do not reproduce it here.

Remark 6.3. Assume there exist $\alpha_{0}, \beta_{0}$ such that

$$
\left\{\begin{array}{l}
\exists\left(\alpha_{0}, \alpha_{1}\right) 1=\frac{f\left(x x_{0}, y y_{0}\right)}{f\left(x_{0}, y_{0}\right)}, \quad \forall(x, y): \frac{\alpha_{0} x+\alpha_{1} y}{\alpha_{0}+\alpha_{1}}=1, \\
1<\frac{f\left(x x_{0}, y y_{0}\right)}{f\left(x_{0}, y_{0}\right)}<2-\frac{\alpha_{0} x+\alpha_{1} y}{\alpha_{0}+\alpha_{1}}, \quad \forall(x, y): \frac{\alpha_{0} x+\alpha_{1} y}{\alpha_{0}+\alpha_{1}}<1, \\
2-\frac{\alpha_{0} x+\alpha_{1} y}{\alpha_{0}+\alpha_{1}}<\frac{f\left(x x_{0}, y y_{0}\right)}{f\left(x_{0}, y_{0}\right)}<1, \quad \forall(x, y): \frac{\alpha_{0} x+\alpha_{1} y}{\alpha_{0}+\alpha_{1}}>1,
\end{array}\right.
$$

with

$$
\frac{\chi N(y)}{N(0)} d y=\left(\alpha_{0} d \mu_{B}(y)+\alpha_{1} d \mu_{\theta}(y)\right) /\left(\alpha_{0}+\alpha_{1}\right) \geq 0,
$$

then the entropy associated to $H(z)=|1-z|$ decreases and we have the global convergence of $n$ to $N$.

REMARK 6.4. The function $f(x, y)=(x+y)^{\omega}$ with $0<\omega<1$ satisfies (6.7) with $\alpha=x_{0} /\left(x_{0}+y_{0}\right)$ and $\beta=y_{0} /\left(x_{0}+y_{0}\right)$. 
6.2. Space and age structured model. Now we consider the following space age model (1.8). We define

$$
d \mu(y, x)=\frac{B(y, x) N(y, x)}{\int_{y^{\prime}=0}^{\infty} B\left(y^{\prime}, x\right) N\left(y^{\prime}, x\right) d y^{\prime}},
$$

and

$$
m_{0}(x)=\int_{y^{\prime}=0}^{\infty} B\left(y^{\prime}, x\right) N\left(y^{\prime}, x\right) d y^{\prime},
$$

where age $y \in[0, \infty[$ and space $x \in]-\infty, \infty[$.

TheOREM 6.5. Assume there exist a positive steady state to the problem (1.8) and its dual

$$
\left\{\begin{array}{l}
\frac{\partial}{\partial y} V N+d(y, x) N(y, x)-\frac{\partial}{\partial x} \nu(y, x) \frac{\partial}{\partial x} N(y, x)=0 \\
V(0, x) N(0, x)=f\left(\int_{0}^{\infty} B\left(y^{\prime}, x\right) N\left(y^{\prime}, x\right) d y^{\prime}\right), \\
\left.\frac{\partial}{\partial x} N\right|_{|x|=\infty}=\left.N\right|_{|x|=\infty}=0, \\
-V(y, x) \frac{\partial}{\partial y} \phi+d(y, x) \phi(y, x)-\frac{\partial}{\partial x} \nu(y, x) \frac{\partial}{\partial x} \phi(y, x)= \\
\quad \phi(0) B(y, x) f\left(\int_{0}^{\infty} B\left(y^{\prime}, x\right) N\left(y^{\prime}, x\right) d y^{\prime}\right) / \int_{0}^{\infty} B\left(y^{\prime}, x\right) N\left(y^{\prime}, x\right) d y^{\prime} .
\end{array}\right.
$$

Then for all positive convex function $H$, we define,

$$
\mathcal{H}(t):=\int_{0}^{\infty} \int_{-\infty}^{\infty} H(n / N) N \phi d y d x
$$

and we have

$$
\begin{aligned}
\frac{d}{d t} \mathcal{H}=\int_{x=-\infty}^{\infty} U(0, x)\left[H\left(\frac{f\left(\int_{y^{\prime}=0}^{\infty} \frac{n\left(t, y^{\prime}, x\right)}{N\left(y^{\prime}, x\right)} d \mu\left(y^{\prime}, x\right) m_{0}(x)\right)}{f\left(m_{0}(x)\right)}\right)\right. \\
\left.\quad-\int_{y=0}^{\infty} H\left(\frac{n}{N}\right) d \mu(y, x)\right] d x \\
\quad-\int_{y=0}^{\infty} \int_{x=-\infty}^{\infty} H^{\prime \prime}(n / N)\left(\partial x \frac{n}{N}\right)^{2} \nu(y, x) N(y, x) \phi(y, x) d y d x
\end{aligned}
$$

where $U(0, x)=N(0, x) V(0, x) \phi(0, x)$. 
Proof. Indeed, proceeding analogously to the theorem 6.1, we have

$$
\begin{aligned}
& \frac{d}{d t} \int_{-\infty}^{\infty} \int_{0}^{\infty} H(n / N) N \phi d y d x=\int_{x=-\infty}^{\infty} N(0, x) V(0, x) \phi(0, x) \\
& {\left[H\left(\frac{f\left(\int_{y^{\prime}=0}^{\infty} B\left(y^{\prime}, x\right) n\left(t, y^{\prime}, x\right) d y^{\prime}\right)}{f\left(\int_{y^{\prime}=0}^{\infty} B\left(y^{\prime}, x\right) N\left(y^{\prime}, x\right) d y^{\prime}\right)}\right)-\frac{\int_{y=0}^{\infty} H(n / N) B(y, x) N(y, x) d y}{\int_{y^{\prime}=0}^{\infty} B\left(y^{\prime}, x\right) N\left(y^{\prime}, x\right) d y^{\prime}}\right] d x} \\
& -\int_{y=0}^{\infty} \int_{x=-\infty}^{\infty} H^{\prime \prime}(n / N)\left(\partial x \frac{n}{N}\right)^{2} \nu(y, x) N(y, x) \phi(y, x) d y d x,
\end{aligned}
$$

then using (6.10), (6.11), we easily complete the proof.

Assumption (3.36) becomes

$$
\left\{\begin{array}{l}
\forall x \geq 0, \quad \forall z>1, \quad 1<f\left(z m_{0}(x)\right) / f\left(m_{0}(x)\right)<z \\
\forall x \geq 0, \quad \forall 0<z<1, \quad 1>f\left(z m_{0}(x)\right) / f\left(m_{0}(x)\right)>z .
\end{array}\right.
$$

Then we have the following property, here we only use the dissipation term associated to the age.

Theorem 6.6. Assume (6.13) and $\int_{-\infty}^{\infty} \int_{0}^{\infty} n\left(0, y^{\prime}, x^{\prime}\right) \phi\left(y^{\prime}, x^{\prime}\right) d y^{\prime} d x^{\prime}>0$, if there exists a solution to (6.12), then

$$
\exists c \in] 0, \infty\left[: \inf _{t \geq 0} \int_{-\infty}^{\infty} \int_{0}^{\infty} n\left(t, y^{\prime}, x^{\prime}\right) \phi\left(y^{\prime}, x^{\prime}\right) d y^{\prime} d x^{\prime} \geq c .\right.
$$

Moreover, if $n(0, .,.) / N(.,.) \in L^{p}\left(\left[0, \infty\left[\times\left[-\infty, \infty\left[, N\left(y^{\prime}, x^{\prime}\right) \phi\left(y^{\prime}, x^{\prime}\right) d y^{\prime} d x^{\prime}\right)\right.\right.\right.\right.$ for some $p \geq 1$ then we have

$$
\lim _{t \rightarrow \infty} n(t, .) / N(.)=1, \quad L^{p}\left(\left[0, \infty\left[\times\left[-\infty, \infty\left[, N\left(y^{\prime}, x^{\prime}\right) \phi\left(y^{\prime}, x^{\prime}\right) d y^{\prime} d x^{\prime}\right) .\right.\right.\right.\right.
$$

THEOREM 6.7. If additionally, we assume that for some $\mu>0$, we have

$$
B(y, x) \geq \alpha \phi(y, x) / \phi(0, x), \quad \forall y, x \geq 0,
$$

and

$$
\begin{aligned}
I=\min \left[1-\sup _{x \in]-\infty, \infty[, z \geq 1}\left(\frac{\frac{f\left(z m_{0}(x)\right)}{f\left(m_{0}(x)\right)}-1}{z-1}\right),\right. & \\
& \left.\inf _{x \in]-\infty, \infty[, z \leq 1}\left(\frac{\frac{f\left(z m_{0}(x)\right)}{f\left(m_{0}(x)\right)}-1}{z-1}\right)-1\right]>0 .
\end{aligned}
$$

Moreover, if $r=I \alpha \inf _{x \geq 0} N(0, x)$ then we obtain

$$
\begin{gathered}
\int_{-\infty}^{\infty} \int_{0}^{\infty}\left|n\left(t, y^{\prime}, x^{\prime}\right)-N\left(y^{\prime}, x^{\prime}\right)\right| \phi\left(y^{\prime}, x^{\prime}\right) d y^{\prime} d x^{\prime} \leq \\
e^{-r t} \int_{-\infty}^{\infty} \int_{0}^{\infty}\left|n\left(0, y^{\prime}, x^{\prime}\right)-N\left(y^{\prime}, x^{\prime}\right)\right| \phi\left(y^{\prime}, x^{\prime}\right) d y^{\prime} d x^{\prime} .
\end{gathered}
$$

The proof of this theorem follows that of theorems 3.9 and 3.10 and we leave it to the reader. The existence proof of $(N, \lambda, \phi)$ solution to $(6.12)$ can be performed using Schauder's fixed point theorem (see $[\mathbf{8}, \mathbf{3}, \mathbf{1 1}])$. 


\section{References}

[1] J.M. Cushing, An Introduction to Structured Population Dynamics, SIAM, Philadelphia, PA, (1998).

[2] O. Diekmann, M. Gyllenberg, H. Huang, M. Kirkilionis, J. A. J. Metz, H. R. Thieme, On the formulation and analysis of general deterministic structured population models. II. Nonlinear theory, J. Math. Biol. 43, no. 2, 2001, p157-189.

[3] M. Escobedo, S. Mischler, M. Rodriguez Ricard, On self-similarity and stationary problem for fragmentation and coagulation models, to appear in Annales IHP (analyse nonlinéaire).

[4] J.Z. Farkas, Stability Conditions for the Non-Linear McKendrick Equations, Applied Mathematics and Computation, 156, 2004, p771-777.

[5] W. Feller. An Introduction to Probability Theory and Its Applications, Volume I. Wiley, New York, 3rd edition, 1968.

[6] M.E. Gurtin, R.C. MacCamy, Non-linear age-dependent populations dynamics, Arch. Rat. Mech. Anal. 54, 1974, p281-300.

[7] M. Iannelli, Mathematical Theory of Age-Structured Population Dynamics, Giardini Editori, Pisa, 1994.

[8] P. Laurençot, Steady states for a fragmentation equation with size diffusion, to appear in a volume of Banach Center Publications.

[9] J. A. J. Metz and O. Diekmann, The dynamics of physiologically structured populations. LN in biomathematics 68, Springer-Verlag, 1986.

[10] P. Michel, PhD Thesis, Univ. Paris 9 Dauphine. In preparation.

[11] P. Michel, Existence of a solution to the cell division eigenproblem. To appear in Math. Mod. Meth. Appl. Sci.

[12] P. Michel, S. Mischler and B. Perthame, General entropy equations for structured population models and scattering, C.R. Acad. Sc. Paris, Sér. I, 338, 2004, p697-702.

[13] P. Michel, S. Mischler and B. Perthame, General relative entropy inequality: an illustration on growth models, J. Math. Pures et Appl. 84, Issue 9, 2005, p1235-1260.

[14] S. Mischler, B. Perthame and L. Ryzhik, Stability in a Nonlinear Population Maturation Model, Math. Models Meth. Appl. Sci., 12, No. 12, 2002, p1751-1772.

[15] B. Perthame and L. Ryzhik, Exponential decay for the fragmentation or cell-division equation. Journal of Differential Equations, 210, Issue 1, March 2005, p155-177.

[16] S. Tuljapurkar, H. Caswell, Structured population models in marine, terrestrial and freshwater ecosystems. Chapman and Hall, New York, New York, USA, 1997.

[17] G.F. Webb, Theory of nonlinear age-dependent population dynamics, Monographs and Textbook in Pure and Applied Mathematics, 89, New-York Basel: Marcel Dekker Inc., 294p, 1985 .

DMA, Ecole Normale Supérieure; 45, Rue d'Ulm, F75230 Paris Cedex 05, FRANCE Current address: DMI, Institut Camille Jordan, Ecole centrale de Lyon; 36 avenue Guy de Collongue, 69134 Ecully

E-mail address: Philippe.Michel@ec-lyon.fr 